\title{
BÄCKLUND TRANSFORMATIONS FOR TRANSPARENT CONNECTIONS
}

\author{
GABRIEL P. PATERNAIN
}

\begin{abstract}
Let $M$ be a closed orientable surface of negative curvature. A connection is said to be transparent if its parallel transport along closed geodesics is the identity. We describe all transparent $S U(2)$-connections and we show that they can be built up from suitable Bäcklund transformations.
\end{abstract}

\section{INTRODUCTION}

Let $(M, g)$ be a closed Riemannian manifold. A unitary connection $\nabla$ on the trivial bundle $M \times \mathbb{C}^{n}$ is said to be transparent if its parallel transport along every closed geodesic of $g$ is the identity. These connections are "ghosts" or "invisible" from the point of view of the closed geodesics of $g$. The problem of determining a connection from its parallel transport along geodesics is a natural integral-geometry problem that can be considered also in the case of manifolds with boundary or $\mathbb{R}^{d}$ with appropriate decay conditions at infinity, and in this context, it has been considered by several authors [1, 2, 9, 12, 13]. It arises for example when one considers the wave equation associated to the Schrödinger equation with external Yang-Mills potential $A$ and the inverse problem of determining the potential $A$ from the Dirichlet-to-Neumann map $\Lambda_{A}$. Returning to the case of closed manifolds we note that recently, L. Mason has classified all transparent connections on $S^{2}$ and $\mathbb{R P}^{2}$ with the standard round metric [7] using a twistor correspondence similar to the one used in [6] to classify anti-selfdual Yang-Mills connections over $S^{2} \times S^{2}$ with split signature.

There is another natural but stronger notion of transparency which arises by considering an appropriate cocycle over the geodesic flow. Write $\nabla=d+A$, where $A: T M \rightarrow \mathfrak{u}(n)$ is a smooth function linear in $v \in T_{x} M$ for all $x \in M$. Consider the geodesic flow $\phi_{t}$ of the metric $g$ acting on the unit sphere bundle $S M$. The connection defines a $U(n)$-valued cocycle over the flow $\phi_{t}$ determined by the ODE of parallel transport along geodesics. In other words, let $C: S M \times \mathbb{R} \rightarrow U(n)$ be given by

$$
\frac{d}{d t} C(x, v, t)=-A\left(\phi_{t}(x, v)\right) C(x, v, t), \quad C(x, v, 0)=\mathrm{Id} .
$$

The function $C$ is a cocycle:

$$
C(x, v, t+s)=C\left(\phi_{t}(x, v), s\right) C(x, v, t)
$$

for all $(x, v) \in S M$ and $s, t \in \mathbb{R}$. The cocycle $C$ is said to be cohomologically trivial if there exists a smooth function $u: S M \rightarrow U(n)$ such that

$$
C(x, v, t)=u\left(\phi_{t}(x, v)\right) u^{-1}(x, v) .
$$


Definition 1.1. We will say that a connection $\nabla$ is cohomologically trivial if $C$ is cohomologically trivial.

Obviously a cohomologically trivial connection is transparent, since the latter simply means $C(x, v, T)=$ Id every time that $\phi_{T}(x, v)=(x, v)$. There is one important situation in which both definitions agree. If $\phi_{t}$ is Anosov, then the Livsic theorem [4, 5] together with the regularity results in [8] imply that a transparent connection is also cohomologically trivial. The Anosov property is satisfied, if for example $(M, g)$ has negative sectional curvature.

In [10] we studied transparent connections over negatively curved surfaces and established several of their basic properties. In the present paper we will explain how to obtain all transparent connections when the structure group is $S U(2)$ (our main result is Theorem 5.4 below). The description of the connections is based on a Bäcklund transformation which is quite reminiscent of Uhlenbeck's work on unitons [11. At the same time we will set up a general correspondence for cohomologically trivial connections over any surface regardless of the sign of its Gaussian curvature thus generalising Theorem B in [10].

Acknowledgement: I am very grateful to Maciej Dunajski for several useful discussions related to this paper. In particular, he suggested to me the possibility that a Bäcklund transformation may exist for transparent connections.

\section{Preliminary Results}

In this section we summarise the setting in [10] and the results needed for the subsequent sections.

Let $M$ be an oriented surface with a Riemannian metric and let $S M$ be its unit tangent bundle. Recall that $S M$ has a canonical framing $\{X, H, V\}$, where $X$ is the geodesic vector field, $V$ is the vertical vector field and $H=[V, X]$ is the horizontal vector field.

Let $\mathbb{M}_{n}(\mathbb{C})$ be the set of $n \times n$ complex matrices. Given functions $u, v: S M \rightarrow$ $\mathbb{M}_{n}(\mathbb{C})$ we consider the inner product

$$
\langle u, v\rangle=\int_{S M} \operatorname{trace}\left(u v^{*}\right) d \mu
$$

where $\mu$ is the Riemannian measure associated with the Sasaki metric of $S M$ which makes $\{X, H, V\}$ into an orthonormal frame. The space $L^{2}\left(S M, \mathbb{M}_{n}(\mathbb{C})\right)$ decomposes orthogonally as a direct sum

$$
L^{2}\left(S M, \mathbb{M}_{n}(\mathbb{C})\right)=\bigoplus_{n \in \mathbb{Z}} H_{n}
$$

where $-i V$ acts as $n$ Id on $H_{n}$.

Following Guillemin and Kazhdan in [3] we introduce the following first order elliptic operators

$$
\eta_{+}, \eta_{-}: C^{\infty}\left(S M, \mathbb{M}_{n}(\mathbb{C})\right) \rightarrow C^{\infty}\left(S M, \mathbb{M}_{n}(\mathbb{C})\right)
$$


given by

$$
\eta_{+}:=(X-i H) / 2, \quad \eta_{-}:=(X+i H) / 2 .
$$

Clearly $X=\eta_{+}+\eta_{-}$. Let $\Omega_{n}:=C^{\infty}\left(S M, \mathbb{M}_{n}(\mathbb{C})\right) \cap H_{n}$. We have

$$
\eta_{+}: \Omega_{n} \rightarrow \Omega_{n+1}, \quad \eta_{-}: \Omega_{n} \rightarrow \Omega_{n-1}, \quad\left(\eta_{+}\right)^{*}=-\eta_{-} .
$$

If a connection $A$ is cohomologically trivial there exists a smooth $u: S M \rightarrow U(n)$ such that $C(x, v, t)=u\left(\phi_{t}(x, v)\right) u^{-1}(x, v)$. Differentiating with respect to $t$ and setting $t=0$, this is equivalent to $X(u)+A u=0$, where we now regard $A$ as a function $A: S M \rightarrow \mathfrak{u}(n)$. To deal with this equation, we introduce the "twisted" operators

$$
\mu_{+}:=\eta_{+}+A_{1}, \quad \mu_{-}:=\eta_{-}+A_{-1} .
$$

where $A=A_{-1}+A_{1}$, and

$$
\begin{gathered}
A_{1}:=\frac{A-i V(A)}{2} \in H_{1}, \\
A_{-1}:=\frac{A+i V(A)}{2} \in H_{-1} .
\end{gathered}
$$

Observe that this decomposition corresponds precisely with the usual decomposition of $\mathfrak{u}(n)$-valued 1 -forms on a surface:

$$
\Omega^{1}(M, \mathfrak{u}(n)) \otimes \mathbb{C}=\Omega^{1,0}(M, \mathfrak{u}(n)) \oplus \Omega^{0,1}(M, \mathfrak{u}(n)),
$$

given by the eigenvalues $\pm i$ of the Hodge star operator $\star$ of the metric $g$.

We also have

$$
\mu_{+}: \Omega_{n} \rightarrow \Omega_{n+1}, \quad \mu_{-}: \Omega_{n} \rightarrow \Omega_{n-1}, \quad\left(\mu_{+}\right)^{*}=-\mu_{-} .
$$

The equation $X(u)+A u=0$ is now $\mu_{+}(u)+\mu_{-}(u)=0$.

Given an element $u \in C^{\infty}\left(S M, \mathbb{M}_{n}(\mathbb{C})\right)$, we write $u=\sum_{m \in \mathbb{Z}} u_{m}$, where $u_{m} \in \Omega_{m}$. We will say that $u$ has degree $N$, if $N$ is the smallest non-negative integer such that $u_{m}=0$ for all $m$ with $|m| \geq N+1$. The following finiteness result will be crucial for us.

Theorem 2.1. [10, Theorem 5.1] If $M$ has negative curvature every solution $u$ of $X(u)+A u=0$ has finite degree.

For future use, it is convenient to write the operators $\eta_{-}$and $\mu_{-}$in local coordinates. Consider isothermal coordinates $(x, y)$ on $M$ such that the metric can be written as $d s^{2}=e^{2 \lambda}\left(d x^{2}+d y^{2}\right)$ where $\lambda$ is a smooth real-valued function of $(x, y)$. This gives coordinates $(x, y, \theta)$ on $S M$ where $\theta$ is the angle between a unit vector $v$ and $\partial / \partial x$. In these coordinates, $V=\partial / \partial \theta$ and the vector fields $X$ and $H$ are given by:

$$
\begin{aligned}
& X=e^{-\lambda}\left(\cos \theta \frac{\partial}{\partial x}+\sin \theta \frac{\partial}{\partial y}+\left(-\frac{\partial \lambda}{\partial x} \sin \theta+\frac{\partial \lambda}{\partial y} \cos \theta\right) \frac{\partial}{\partial \theta}\right) \\
& H=e^{-\lambda}\left(-\sin \theta \frac{\partial}{\partial x}+\cos \theta \frac{\partial}{\partial y}-\left(\frac{\partial \lambda}{\partial x} \cos \theta+\frac{\partial \lambda}{\partial y} \sin \theta\right) \frac{\partial}{\partial \theta}\right) .
\end{aligned}
$$


Consider $u \in \Omega_{n}$ and write it locally as $u(x, y, \theta)=h(x, y) e^{i n \theta}$. Using these formulas a simple, but tedious calculation shows that

$$
\eta_{-}(u)=e^{-(1+n) \lambda} \bar{\partial}\left(h e^{n \lambda}\right) e^{i(n-1) \theta},
$$

where $\bar{\partial}=\frac{1}{2}\left(\frac{\partial}{\partial x}+i \frac{\partial}{\partial y}\right)$. In order to write $\mu_{-}$suppose that $A(x, y, \theta)=a(x, y) \cos \theta+$ $b(x, y) \sin \theta$. If we also write $A=A_{x} d x+A_{y} d y$, then $A_{x}=a e^{\lambda}$ and $A_{y}=b e^{\lambda}$. Let $A_{\bar{z}}:=\frac{1}{2}\left(A_{x}+i A_{y}\right)$. Using the definition of $A_{-1}$ we derive

$$
A_{-1}=\frac{1}{2}(a+i b) e^{-i \theta}=A_{\bar{z}} d \bar{z} .
$$

Putting this together with (11) we obtain

$$
\mu_{-}(u)=e^{-(1+n) \lambda}\left(\bar{\partial}\left(h e^{n \lambda}\right)+A_{\bar{z}} h e^{n \lambda}\right) e^{i(n-1) \theta}
$$

Note that $\Omega_{n}$ can be identified with the set of smooth sections of the bundle $(M \times$ $\left.\mathbb{M}_{2}(\mathbb{C})\right) \otimes K^{\otimes n}$ where $K$ is the canonical line bundle. The identification takes $u=h e^{i n \theta}$ into $h e^{n \lambda}(d z)^{n}(n \geq 0)$ and $u=h e^{-i n \theta} \in \Omega_{-n}$ into $h e^{n \lambda}(d \bar{z})^{n}$. The second equality in (2) should be understood using this identification.

\section{A GenERAL CORRESPONDENCE}

The purpose of this section is to briefly indicate that the calculations done in the proof of Theorem B in [10] also give a general classification result for cohomologically trivial connections on any surface.

Let $M$ be an oriented surface with a Riemannian metric and let $S M$ be its unit tangent bundle. Let

$$
\mathcal{A}:=\left\{A: S M \rightarrow \mathfrak{u}(n): V^{2}(A)=-A\right\} .
$$

The set $\mathcal{A}$ is identified with the set of all unitary connections on the trivial bundle $M \times \mathbb{C}^{n}$. Indeed, a function $A$ satisfying $V^{2}(A)+A=0$ is a function that locally can be written as $A(x, y, \theta)=a(x, y) \cos \theta+b(x, y) \sin \theta$.

Recall from the introduction that $A$ is said to be cohomologically trivial if there exists a smooth $u: S M \rightarrow U(n)$ such that $C(x, v, t)=u\left(\phi_{t}(x, v)\right) u^{-1}(x, v)$. Differentiating with respect to $t$ and setting $t=0$ this is equivalent to

$$
X(u)+A u=0 .
$$

Let $\mathcal{A}_{0}$ be the set of all cohomologically trivial connections, that is, the set of all $A \in \mathcal{A}$ such that there exists $u: S M \rightarrow U(n)$ for which (4) holds.

Given a vector field $W$ in $S M$, let $G_{W}$ be the set of all $u: S M \rightarrow U(n)$ such that $W(u)=0$, i.e. first integrals of $W$. Note that $G_{V}$ is nothing but the group of gauge transformations of the trivial bundle $M \times \mathbb{C}^{n}$.

We wish to understand $\mathcal{A}_{0} / G_{V}$. Now let $\mathcal{H}_{0}$ be the set of all $f: S M \rightarrow \mathfrak{u}(n)$ such that

$$
H(f)+V X(f)=[X(f), f]
$$

and there is $u: S M \rightarrow U(n)$ such that $f=u^{-1} V(u)$. It is easy to check that $G_{X}$ acts on $\mathcal{H}_{0}$ by $f \mapsto a^{-1} f a+a^{-1} V(a)$ where $a \in G_{X}$. 
Theorem 3.1. There is a 1-1 correspondence between $\mathcal{A}_{0} / G_{V}$ and $\mathcal{H}_{0} / G_{X}$.

Proof. Forward direction: a cohomologically trivial connection $A$ comes with a $u$ such that $X(u)+A u=0$. If we set $f:=u^{-1} V(u)$, then $f \in \mathcal{H}_{0}$, i.e., $f$ satisfies the PDE $H(f)+V X(f)=[X(f), f]$. This is a calculation, exactly as in the proof of Theorem $\mathrm{B}$ in [10], but for the reader's convenience we explain the geometric origin of this equation. Using $u$ we may define a connection on $S M$ gauge equivalent to $\pi^{*} A$ by setting $B:=u^{-1} d u+u^{-1} \pi^{*} A u$, where $\pi: S M \rightarrow M$ is the foot-point projection. Since $\pi^{*} A$ is the pull-back of a connection on $M$, the curvature $F_{B}$ of $B$ must vanish when one of the entries is the vertical vector field $V$. The PDE $H(f)+V X(f)=[X(f), f]$ arises by combining the two equations $F_{B}(X, V)=F_{B}(H, V)=0$ with $B(X)=0$.

Backward direction: Given $f$ with $f u=V(u)$, set $A:=-X(u) u^{-1}$. Then $A \in \mathcal{A}_{0}$, i.e. $V^{2}(A)=-A$; again this is a calculation done fully in Theorem $\mathrm{B}$ in [10].

Now there are two ambiguities here. Going forward, we may change $u$ as long as we solve $X(u)+A u=0$. This changes $f$ by the action of $G_{X}$. Going backwards we may change $u$ as long as $f u=V(u)$, this changes $A$ by a gauge transformation, i.e. an element in $G_{V}$.

Remark 3.2. Note that if the geodesic flow is transitive (i.e. there is a dense orbit) the only first integrals are the constants and thus $G_{X}=U(n)$ acts simply by conjugation. If $M$ is closed and of negative curvature, the geodesic flow is Anosov and therefore transitive.

The fact that the PDE describing cohomologically trivial connections arises from zero curvature conditions is an indication of the possible "integrable" nature of the problem at hand. The existence of a Bäcklund transformation that we will introduce shortly is another typical feature of integrable systems. An interesting point here is that the space $\mathcal{H}_{0} / G_{X}$ is in some sense simpler and larger when the underlying geodesic flow is more complicated, i.e. when it is transitive $G_{X}$ reduces to $U(n)$.

\section{THE BÄCKLUND TRANSFORMATION}

For the remainder of this paper we restrict to the case in which the structure group is $S U(2)$.

Suppose there is a smooth map $b: S M \rightarrow S U(2)$ such that $f:=b^{-1} V(b)$ solves the PDE:

$$
H(f)+V X(f)=[X(f), f] .
$$

Then, by Theorem $3.1, A:=-X(b) b^{-1}$ defines a cohomologically trivial connection on $M$ and $-\star A=V(A)=-b X(f) b^{-1}-H(b) b^{-1}$.

Lemma 4.1. Let $g: M \rightarrow \mathfrak{s u}(2)$ be a smooth map with $\operatorname{det} g=1$ (i.e. $\left.g^{2}=-\mathrm{Id}\right)$. Then, there exists $a: S M \rightarrow S U(2)$ such that $g=a^{-1} V(a)$.

Proof. Let $L(x)$ (resp. $U(x)$ ) be the eigenspace corresponding to the eigenvalue $i$ (resp. $-i$ ) of $g(x)$. We have an orthogonal decomposition $\mathbb{C}^{2}=L(x) \oplus U(x)$ for every $x \in M$. Consider sections $\alpha \in \Omega^{1,0}(M, \mathbb{C})$ and $\beta \in \Omega^{1,0}(M, \operatorname{Hom}(L, U))=$ 
$\Omega^{1,0}\left(M, L^{*} U\right)$ such that $|\alpha|^{2}+|\beta|^{2}=1$. Such a pair of sections always exists; for example, we can choose a section $\tilde{\beta}$ with a finite number of isolated zeros and then choose $\tilde{\alpha}$ such that it does not vanish on the zeros of $\tilde{\beta}$. Then we set $\alpha:=\tilde{\alpha} /\left(|\tilde{\alpha}|^{2}+|\tilde{\beta}|^{2}\right)^{1 / 2}$ and $\beta:=\tilde{\beta} /\left(|\tilde{\alpha}|^{2}+|\tilde{\beta}|^{2}\right)^{1 / 2}$. Note that $\bar{\alpha} \in \Omega^{0,1}(M, \mathbb{C})$ and $\beta^{*} \in \Omega^{0,1}(M, \operatorname{Hom}(U, L))=$ $\Omega^{0,1}\left(M, U^{*} L\right)$. Using the orthogonal decomposition we define $a: S M \rightarrow S U(2)$ by

$$
a(x, v)=\left(\begin{array}{cc}
\alpha(x, v) & \beta^{*}(x, v) \\
-\beta(x, v) & \bar{\alpha}(x, v)
\end{array}\right) .
$$

Clearly $a=a_{-1}+a_{1}$, where

$$
a_{1}=\left(\begin{array}{cc}
\alpha & 0 \\
-\beta & 0
\end{array}\right)
$$

and

$$
a_{-1}=\left(\begin{array}{cc}
0 & \beta^{*} \\
0 & \bar{\alpha}
\end{array}\right) .
$$

It is straightforward to check that $a g=V(a)$.

Now let $u:=a b: S M \rightarrow S U(2)$ and let $F:=(a b)^{-1} V(a b)=b^{-1} g b+f$.

Question. When does $F$ satisfy (15)?

If it does, then it defines (via Theorem 3.1) a new cohomologically trivial connection given by

$$
A_{F}=-X(a b)(a b)^{-1}=-X(a) a^{-1}+a A a^{-1},
$$

where $A$ is the cohomologically trivial connection associated to $f$.

Recall that the connection $A$ defines a covariant derivative $d_{A} g=d g+[A, g]$.

Lemma 4.2. F satisfies (5) if and only if

$$
-\star d_{A} g=\left(d_{A} g\right) g \text {. }
$$

Proof. Starting with $F=b^{-1} g b+f$ and using that $A=-X(b) b^{-1}=b X\left(b^{-1}\right)$ we compute

$$
X(F)=b^{-1}([A, g]+X(g)) b+X(f) .
$$

Similarly, using $H(b)=(\star A) b-b X(f)$ we find

$$
H(F)=b^{-1}([-\star A, g]+H(g)) b+\left[X(f), b^{-1} g b\right]+H(f) .
$$

Now we compute $V X(F)$; here we use that $V(g)=0$. We obtain

$$
V X(F)=\left[b^{-1}([A, g]+X(g)) b, f\right]+b^{-1}([-\star A, g]+V X(g)) b+V X(f) .
$$

The last term we need for (5) is:

$$
[X(F), F]=b^{-1}[[A, g]+X(g), g] b+\left[b^{-1}([A, g]+X(g)) b, f\right]+\left[X(f), b^{-1} g b\right]+[X(f), f] \text {. }
$$

Since $f$ satisfies (5) we see that $F$ satisfies (5) if and only if

$$
H(g)+V X(g)-2[\star A, g]=[[A, g]+X(g), g] .
$$


Since $g$ depends only on the base point and $g^{2}=-$ Id we can rewrite this as

$$
-2 \star(d g+[A, g])=[d g+[A, g], g]=2(d g+[A, g]) g .
$$

Thus $F$ satisfies (5) if and only if

$$
-\star d_{A} g=\left(d_{A} g\right) g
$$

as claimed.

We will now rephrase equation (6) in terms of holomorphic line bundles. Recall that the connection $A$ induces a holomorphic structure on the trivial bundle $M \times \mathbb{C}^{2}$ and on the endomorphism bundle $M \times \mathbb{M}_{2}(\mathbb{C})$. We have an operator $\bar{\partial}_{A}=\left(d_{A}-i \star d_{A}\right) / 2=$ $\bar{\partial}+\left[A_{-1}, \cdot\right]$ acting on sections $f: M \rightarrow \mathbb{M}_{2}(\mathbb{C})$.

Set $\pi:=(\mathrm{Id}-i g) / 2$ and $\pi^{\perp}=(\mathrm{Id}+i g) / 2$ so that $\pi+\pi^{\perp}=\mathrm{Id}$. Let $L(x)$ be as above the eigenspace corresponding to the eigenvalue $i$ of $g(x)$. Note that $\pi$ is the Hermitian orthogonal projection over $L(x)=\operatorname{Image}(\pi(x))$.

Lemma 4.3. Let $g: M \rightarrow \mathfrak{s u}(2)$ be a smooth map with $\operatorname{det} g=1$. The following are equivalent:

(1) $-\star d_{A} g=\left(d_{A} g\right) g$;

(2) $L$ is a $\bar{\partial}_{A}$-holomorphic line bundle;

(3) $\pi^{\perp} \bar{\partial}_{A} \pi=0$.

Proof. Suppose that (1) holds. Apply $\star$ to obtain: $d_{A} g=\left(\star d_{A} g\right) g$. Thus $d_{A} g-i \star$ $d_{A} g=i\left(d_{A} g-i \star d_{A} g\right) g$. In other words $\bar{\partial}_{A} g=i\left(\bar{\partial}_{A} g\right) g=-i g\left(\bar{\partial}_{A} g\right)$ (recall that $\left.g^{2}=-\mathrm{Id}\right)$. Since $\pi=(\mathrm{Id}-i g) / 2$, then $\bar{\partial}_{A} g=-i g\left(\bar{\partial}_{A} g\right)$ is equivalent to $\pi^{\perp} \bar{\partial}_{A} \pi=0$ which is (3).

Using the condition $\pi^{2}=\pi$, we see that $\pi^{\perp} \bar{\partial}_{A} \pi=0$ is equivalent to $\left(\bar{\partial}_{A} \pi\right) \pi=0$. The line bundle $L$ is holomorphic iff given a local section $\xi$ of $L$, then $\bar{\partial}_{A} \xi \in L$. Using that $\pi \xi=\xi$ we see that $\bar{\partial}_{A} \xi \in L$ iff $\left(\bar{\partial}_{A} \pi\right) \xi=0$. Clearly, this happens iff $\left(\bar{\partial}_{A} \pi\right) \pi=0$ and thus (2) holds iff (3) holds.

The next theorem summarises the Bäcklund transformation introduced in this section and it follows directly from Lemmas 4.2 and 4.3 and Theorem 3.1 .

Theorem 4.4. Let $A$ be a cohomologically trivial connection and let $L$ be a holomorphic line subbundle of the trivial bundle $M \times \mathbb{C}^{2}$ with respect to the complex structure induced by $A$. Define a map $g: M \rightarrow \mathfrak{s u}(2)$ with $\operatorname{det} g=1$ by declaring $L$ to be its eigenspace with eigenvalue $i$. Consider $a: S M \rightarrow S U(2)$ with $g=a^{-1} V(a)$ as given by Lemma 4.1. Then

$$
A_{F}:=-X(a) a^{-1}+a A a^{-1}
$$

defines a cohomologically trivial connection.

Remark 4.5. Note that if the geodesic flow is transitive, two solutions $u, w$ of $X(u)+A u=0$ are related by $u=w g$ where $g$ is a constant unitary matrix, because $X\left(w^{-1} u\right)=0$. Thus the degrees of $u$ and $w$ are the same. We can then 
talk about the "degree" of a cohomologically trivial connection as the degree of any solution of $X(u)+A u=0$.

If we start, for example, with the trivial connection $A=0$ (which is obviously transparent), then a map $g: M \rightarrow \mathfrak{s u}(2)$ with $\operatorname{det} g=1$ and $-\star d g=(d g) g$ can be identified with a meromorphic function. The connections of degree one $A_{F}=$ $-X(a) a^{-1}$ given by Theorem 4.4 were found in [10]. To some extent, the point of this paper is to show that one can continue this process and also run it "backwards" to decrease the degree of a transparent connection. In the next section we will show that any cohomologically trivial connection such that the associated $u$ has a finite Fourier series can be built up by successive applications of the transformation described in Theorem 4.4, provided that the geodesic flow is transitive.

\section{LOWERING DEGREE USING BÄCKLUND TRANSFORMATIONS}

Let $A$ be a transparent connection with $A=-X(b) b^{-1}$ and $f=b^{-1} V(b)$, where $b: S M \rightarrow S U(2)$, is as in the previous section.

We first make some remarks concerning the $S U(2)$-structure. Let $j: \mathbb{C}^{2} \rightarrow \mathbb{C}^{2}$ be the antilinear map given by

$$
j\left(z_{1}, z_{2}\right)=\left(-\bar{z}_{2}, \bar{z}_{1}\right)
$$

If we think of a matrix $a \in S U(2)$ as a linear map $a: \mathbb{C}^{2} \rightarrow \mathbb{C}^{2}$, then $j a=a j$. This implies that given $b: S M \rightarrow S U(2)$ with $b=\sum_{k \in \mathbb{Z}} b_{k}$, then $j b_{k}=b_{-k} j$ for all $k \in \mathbb{Z}$.

Assumption. Suppose $b$ has a finite Fourier expansion, i.e., $b=\sum_{k=-N}^{k=N} b_{k}$, where $N \geq 1$. By Theorem 2.1 we know that this holds if $M$ has negative curvature.

Let us assume also that $N$ is the degree of $b$ and thus both $b_{N}$ and $b_{-N}=-j b_{N} j$ are non-zero.

The unitary condition $b b^{*}=b^{*} b=$ Id implies that $b_{N} b_{-N}^{*}=b_{-N}^{*} b_{N}=0$. These relations imply that the rank of $b_{-N}$ and $b_{N}$ is at most one and equals one on an open set, which, as we will see shortly, must be all of $M$ except for perhaps a finite number of points.

Consider now a fixed vector $\xi \in \mathbb{C}^{2}$ such that $s(x, v):=b_{-N}(x, v) \xi \in \mathbb{C}^{2}$ is not zero identically. Clearly $s$ can be seen as a section of $\left(M \times \mathbb{C}^{2}\right) \otimes K^{\otimes-N}$. We may write $b_{-N}$ in local isothermal coordinates as $b_{-N}=h e^{-i N \theta}$, using the notation from Section 2. We can thus write $s$ locally as $s=e^{N \lambda} h \xi(d \bar{z})^{N}$.

Lemma 5.1. The local section $e^{-2 N \lambda} s$ is $\bar{\partial}_{A}$-holomorphic.

Proof. Using the operators $\mu_{ \pm}$introduced in Section 2 we can write $X(b)+A b=0$ as

$$
\mu_{+}\left(b_{k-1}\right)+\mu_{-}\left(b_{k+1}\right)=0
$$

for all $k$. This gives $\mu_{+}\left(b_{N}\right)=\mu_{-}\left(b_{-N}\right)=0$. But $\mu_{-}\left(b_{-N}\right)=0$ is saying that $e^{-2 N \lambda} s$ is $\bar{\partial}_{A}$-holomorphic. Indeed, using (3), we see that $\mu_{-}\left(b_{-N}\right)=0$ implies

$$
\bar{\partial}\left(h e^{-N \lambda}\right)+A_{\bar{z}} h e^{-N \lambda}=0
$$


which in turn implies

$$
\bar{\partial}\left(e^{-N \lambda} h \xi\right)+A_{\bar{z}} e^{-N \lambda} h \xi=0 .
$$

This equation says that $e^{-2 N \lambda} s=e^{-N \lambda} h \xi(d \bar{z})^{N}$ is $\bar{\partial}_{A^{-}}$holomorphic.

The section $s$ spans a line bundle $L$ over $M$ which by the previous lemma is $\bar{\partial}_{A^{-}}$ holomorphic. The section $s$ may have zeros, but at a zero $z_{0}$, the line bundle extends holomorphically. Indeed, in a neighbourhood of $z_{0}$ we may write $e^{-2 N \lambda(z)} s(z)=$ $\left(z-z_{0}\right)^{k} w(z)$, where $w$ is a local holomorphic section with $w\left(z_{0}\right) \neq 0$. The section $w$ spans a holomorphic line subbundle which coincides with the one spanned by $s$ off $z_{0}$. Therefore $L$ is a $\bar{\partial}_{A}$-holomorphic line bundle that contains the image of $b_{-N}$ (and $U=j L$ is an anti-holomorphic line bundle that contains the image of $b_{N}$ ). We summarise this in a lemma:

Lemma 5.2. The line bundle $L$ determined by the image of $b_{-N}$ is $\bar{\partial}_{A}$-holomorphic.

We now wish to use the line bundle $L$ to construct an appropriate $g: M \rightarrow \mathfrak{s u}(2)$ such that when we run the Bäcklund transformation from the previous section we obtain a cohomologically trivial connection of degree $\leq N-1$. But first we need the following lemma. Recall that a matrix-valued function $f: S M \rightarrow \mathbb{M}_{n}(\mathbb{C})$ is said to be odd if $f(x, v)=-f(x,-v)$ and even if $f(x, v)=f(x,-v)$.

Lemma 5.3. Assume that the geodesic flow is transitive and let $b: S M \rightarrow S U(2)$ solve $X(b)+A b=0$. Then $b$ is either even or odd.

Proof. Write $b=b_{o}+b_{e}$ where $b_{o}$ is odd and $b_{e}$ is even. Since the operator $(X+A)$ maps even to odd and odd to even, the equation $X(b)+A b=0$ decouples as

$$
\begin{aligned}
& X\left(b_{o}\right)+A b_{o}=0 ; \\
& X\left(b_{e}\right)+A b_{e}=0 .
\end{aligned}
$$

A calculation using these equations shows that $X\left(b_{o}^{*} b_{o}\right)=X\left(b_{e}^{*} b_{e}\right)=X\left(b_{o}^{*} b_{e}\right)=0$. Since the geodesic flow is transitive, these matrices are all constant. Moreover, since $b_{o}^{*} b_{e}$ is odd it must be zero. On the other hand $j b=b j$ implies that $j b_{o}=b_{o} j$ and $j b_{e}=b_{e} j$, which in turn implies that both $b_{o}$ and $b_{e}$ cannot have rank 1 . Putting all this together, we see that either $b_{o}$ or $b_{e}$ must vanish identically.

Suppose that the geodesic flow is transitive. By Lemma 5.3, $b=b_{-N}+d+b_{N}$, where $d$ has degree $\leq N-2$. We now seek $a: S M \rightarrow S U(2)$ of degree one such that $u:=a b$ has degree $\leq N-1$. For this we need $a_{1} b_{N}=a_{-1} b_{-N}=0$. We take a map $g: M \rightarrow \mathfrak{s u}(2)$ with $\operatorname{det} g=1$ such that its $i$ eigenspace is $L$ and its $-i$ eigenspace is $U$. By Lemmas 4.3 and $5.2,-\star d_{A} g=\left(d_{A} g\right) g$. The construction of $a$ with $a g=V(a)$ from Lemma 4.1 is precisely such that the kernel of $a_{-1}$ is $L$ and the kernel of $a_{1}$ is $U$, so the needed relations to lower the degree hold.

Finally by Theorem 4.4, $u$ gives rise to a cohomologically trivial connection $-X(u) u^{-1}$. Combining this with Theorem 2.1 we have arrived at the main result of this paper: 
Theorem 5.4. Let $M$ be a closed orientable surface of negative curvature. Then any transparent $S U(2)$-connection can be obtained by successive applications of Bäcklund transformations as described in Theorem 4.4.

Remark 5.5. It should be possible to do a similar analysis when the structure group is $U(n)$ by considering maps $g: M \rightarrow G_{k, n}$ where $G_{k, n}$ is the complex Grassmannian of $k$-planes in $\mathbb{C}^{n}$. There is a natural embedding of $G_{k, n}$ into $U(n)$ defined by sending a $k$-plane $\ell \in G_{k, n}$ into the unitary map $i\left(\pi_{\ell}-\pi_{\ell^{\perp}}\right)$, where $\pi_{\ell}$ denotes the Hermitian orthogonal projection onto $\ell$. It would also be interesting to understand the set of transparent connections for the case of non-trivial bundles and for structure groups other than $U(n)$.

\section{REFERENCES}

[1] G. Eskin, On non-abelian Radon transform, Russ. J. Math. Phys. 11 (2004) 391-408.

[2] D. Finch, G. Uhlmann, The X-ray transform for a non-abelian connection in two dimensions, Inverse Problems 17 (2001) 695-701.

[3] V. Guillemin, D. Kazhdan, Some inverse spectral results for negatively curved 2-manifolds, Topology 19 (1980) 301-312.

[4] A.N. Livsic, Certain properties of the homology of $Y$-systems, Mat. Zametki 10 (1971) 555-564.

[5] A.N. Livsic, Cohomology of dynamical systems, Izv. Akad. Nauk SSSR Ser. Mat. 36 (1972) 1296-1320.

[6] L.J. Mason, Global anti-self dual Yang-Mills fields in split signature and their scattering, J. Reine Angew. Math. 597 (2006) 105-133.

[7] L.J. Mason, Lecture at Cambridge, November $200 \%$.

[8] V. Niţică, A. Török, Regularity of the transfer map for cohomologous cocycles, Ergodic Theory Dynam. Systems 18 (1998) 1187-1209.

[9] R. Novikov, On determination of a gauge field on $\mathbb{R}^{d}$ from its non-abelian Radon transform along oriented straight lines, J. Inst. Math. Jussieu 1 (2002) 559-629.

[10] G.P. Paternain, Transparent connections over negatively curved surfaces, J. Mod. Dyn. 3 (2009) $311-333$.

[11] K. Uhlenbeck, Harmonic maps into Lie groups: classical solutions of the chiral model, J. Differential Geom. 30 (1989) 1-50.

[12] V.A. Sharafutdinov, On an inverse problem of determining a connection on a vector bundle, J. Inverse and Ill-Posed Problems 8 (2000) 51-88.

[13] L.B. Vertgeim, Integral geometry with a matrix weight, and a nonlinear problem of recovering matrices, Sov. Math.-Dokl. 44 (1992) 132-135.

Department of Pure Mathematics and Mathematical Statistics, University of Cambridge, Cambridge CB3 0WB, UK

E-mail address: g.p.paternain@dpmms.cam.ac.uk 